Revista de Psicología de la PUCP. Vol. XVI, 2, 1998.

\title{
LLANTO DEL ADULTO
}

\author{
Alegría Majluf \\ Universidad Peruana Cayetano Heredia
}

Se presentan los resultados de un primer estudio transcultural del llanto del adulto que comprende 30 países, entre los que se encuentra el Perú. Vingerhoets et al. (1997) de la Universidad de Tilburg, Holanda, analizan las causas, tendencia y frecuencia del llanto, emociones y sentimientos que inducen al llanto, diferencias y similitudes entre los países y los sexos. Los autores encuentran que las mujeres tienden a llorar con más frecuencia e intensidad que los varones, advirtiendo que en algunos países las mujeres establecen mayor relación entre la tendencia al llanto y el ciclo menstrual que en orros.

Palabras claves: transcultural, llanto del adulto.

The results of a first cross-cultural study on adult crying in 30 countries including Peru are presented. Vingerhoets et al. (1997) from the University of Tilburg have analyzed motives, tendencies and frequency of crying, emotions and feelings that provoke crying, as well as differences and similaritics among countries and sexes. Results show that women have a tendency to cry more frequently and intensively than men. It has also been noted thar, in certain countries, there is a link between crying in women and their monthly period.

Key words: cross-cultural, adult crying

1. Obruvo el tírulo de bachiller en Psicología en la Universidad Nacional Mayor de San Marcos. Realizó una maestría sobre desarrollo infantil en la Universidad Estatal de Iowa. Obtuvo el doctorado en el California School of Professional Psychology. Ha realizado estudios sobre el desarrollo de los niños en zonas urbano-marginales, centrándose en la capacidad cognitiva y el léxico. Actualmente trabaja en un centro de beneficencia en una zona urbano-marginal en Chorrillos. Dirección: J. Quiñones 175 - Miraflores. 



\section{Introducción}

El llanto es una expresión emocional típicamente humana, una respuesta de todo el organismo en su complejidad fisiológica, cognitiva y socioemocional que ha recibido poca atención de las ciencias de la conducta.

Una revisión de la literatura conducida por Cornelius y colaboradores (1986) de 70 artículos de revistas americanas e inglesas, entre 1848 a 1989, arrojó escasa información respecto al llanto, aunque, se encontró que se habian postulado algunas teorías, entre estas: la ley Frey (1985) a quien se le considera el pionero de la investigación actual del llanto, quien como bioquímico, considera que el llanto tenía por fin liberar al organismo de sustancias tóxicas y reducir la tensión. Kottler (1996) pensaba que el llanto era una defensa compensatoria contra impulsos como la agresión o el impulso sexual. Frijda (1986) veía el llanto como un signo de impotencia, y carencia de poder y Cornelius $(1981,1987)$ enfatizaba el aspecto social y la interacción con el ambiente. Para este autor el llanto sería un medio de expresión, un medio para despertar simpatías, ayuda, lástima o para manipular a otros.

Las teorías mencionadas, aunque eran de interés, no habían sido objeto de estudios sistemáticos para corroborarlas. Asimismo, los datos reportados acerca del llanto no eran confiables, ya que eran el resultado de estudios poco sistemáticos y carentes de fundamentación teórica, por lo que Vingerhoets y colaboradores en 1985 realizaron una primera investigación sistemática, aunque de carácter exploratorio, respecto a los aspectos psicológicos del llanto. 
El estudio estuvo basado en el trabajo de Scherer, Summerfield y Wallbott (1983), cuyo interés especial era conocer las situaciones especificas que inducen al llanto, la asociación con el género, con la personalidad, con los factores económicos, socioculturales, así como, conocer las consecuencias del llanto para la persona y su ambiente.

Posteriormente Vingerhoets y su grupo, basados parcialmente en los resultados obtenidos en el estudio citado, desarrollaron un modelo del llanto de los adultos (Apéndice) y tanto los resultados como el modelo sirvieron de base para elaborar un cuestionario que en aquella época consideraron que ya era oportuno utilizarlo en un estudio transcultural sobre el llanto de los adultos.

Para realizar dicho estudio los autores contactaron investigadores de diferentes países. Al inicio, 30 investigadores se compromerieron a administrar el cuestionario a 50 varones y 50 mujeres en cada país, aunque, el número varió de país a país. Como se constata en el Cuadro 1, Perú también participó.

\section{Metodología}

\section{Participantes}

Se eligió al azar a estudiantes universitarios y se sugirió equilibrar la cantidad entre ambos géneros, lo cual no siempre fue posible. La muestra de los 30 países estuvo construida por 3967 personas, 2280 mujeres y 1687 hombres (Cuadro 1). Se debe recalcar que no todos ellos respondieron a todos los cuestionarios, reduciéndose la muestra en algunos análisis. La edad promedio fue 23 años.

\section{Instrumentos}

Se administró el Cuestionario del Llanto del Adulto basado en la Escala de Afrontamiento del llanto de Labott y Martin (1987) revisado y ampliado por Vingerhoets. Dicho cuestionario consta de varias partes. 200 
El llanto del adulto

Cuadro 1

Caracteristicas de la Muestra

\begin{tabular}{|c|c|c|c|}
\hline 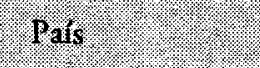 & Wugres & Varanes & Total \\
\hline Australia & 183 & 67 & 250 \\
\hline Austria & 50 & 30 & 80 \\
\hline Bélgica & 171 & 55 & 227 \\
\hline Brasil & 53 & 50 & 103 \\
\hline Bulgaria & 51 & 50 & 101 \\
\hline Chile & 55 & 53 & 108 \\
\hline China & 39 & 38 & 77 \\
\hline Finlandia & 49 & 56 & 105 \\
\hline Ghana & 53 & 54 & 107 \\
\hline Grecia & 79 & 40 & 119 \\
\hline India & 114 & 167 & 281 \\
\hline Indonesia & 118 & 54 & 172 \\
\hline Israel & 50 & 50 & 100 \\
\hline Italia & 79 & 38 & 117 \\
\hline Jamaica & 64 & 36 & 100 \\
\hline Kenya & 82 & 85 & 167 \\
\hline Lituania & 135 & 61 & 196 \\
\hline Malasia & 48 & 43 & 91 \\
\hline Holanda & 79 & 92 & 171 \\
\hline Nueva Zelanda & 49 & 28 & 77 \\
\hline Nigeria & 41 & 58 & 99 \\
\hline Perú & 48 & 45 & 93 \\
\hline Polonia & 56 & 53 & 109 \\
\hline Portugal & 64 & 63 & 127 \\
\hline Rumania & 92 & 81 & 173 \\
\hline España & 51 & 50 & 101 \\
\hline Suecia & 49 & 51 & 100 \\
\hline Suiza & 67 & 34 & 101 \\
\hline Turquía & 52 & 50 & 102 \\
\hline Estados Unidos & 160 & 53 & 213 \\
\hline Total & 2280 & 1687 & 967 \\
\hline
\end{tabular}


El presente artículo se limitará a describir las secciones A, D y E cuyos resultados fueron analizados inicialmente por el autor. El análisis de las otras secciones se encuentra actualmente en proceso. La parte A evalúa la tendencia y frecuencia del llanto. Está conformada por 59 items. Los primeros 55 items constituyen un inventario del llanto, éstos enumeran las situaciones o emociones que inducen al llanto, derivándose de esta sección el Puntaje Total del Llanto. Los items 56 al 59 estiman si ha habido cambios en la tendencia o frecuencia del llanto y los sentimientos después del episodio del llanto. La parte D incluye preguntas respecto al último episodio del llanto en el sujeto y el contexto. La sección $\mathrm{E}$ analiza la relación posible entre la tendencia al llanto y la etapa del ciclo menstrual.

\section{Resultados}

Como se indicó anteriormente, Vingerhcets analizó primero las secciones $\mathrm{A}, \mathrm{D}$ y $\mathrm{E}$, cuyos resultados fueron comunicados a todos los investigadores que participaron en el estudio, enviando así mismo, a cada investigador los datos de sus respectivos países para ser analizados y publicados si así lo desearan.

Considerando la importancia de este primer estudio sistemático respecto al llanto, la autora del presente artículo, quien también colaboró en la investigación pensó que sería interesante dar a conocer en el país los resultados preliminares logrados por Vingerhoets en los 30 países, entre los que se encuentra el Perú. A continuación se describirán los resultados de los 30 países.

\section{Parte $A$}

\section{¿Con qué frecuencia lloramos?}

En el curso de 4 semanas, antes de llenar el cuestionario, 55\% de los varones y $17 \%$ de las mujeres reportaron que no habían llorado. Se observó en la población de 30 países, que las mujeres lloraban con más frecuencia 
El llanto del adulto

Cuadro 2

Frecuencia Estimada del Llanto en 4 Semanas

\begin{tabular}{|c|c|c|}
\hline Pals : & Mujeres & Varones \\
\hline Australia & 2.75 & 1.44 \\
\hline Austria & 2.59 & 1.86 \\
\hline Bélgica & 3.02 & 1.13 \\
\hline Brasil & 2.57 & 0.75 \\
\hline Bulgaria & 1.75 & 0.43 \\
\hline Chile & 3.56 & 1.22 \\
\hline China & 1.26 & 0.39 \\
\hline Finlandia & 3.13 & 1.38 \\
\hline Ghana & 1.76 & 0.62 \\
\hline Grecia & 2.95 & 1.03 \\
\hline India & 2.31 & 0.94 \\
\hline Indonesia & 2.08 & 0.75 \\
\hline Israel & 2.74 & 1.30 \\
\hline Italia & 3.23 & 1.68 \\
\hline Jamaica & 2.13 & 1.21 \\
\hline Kenya & 2.13 & 1.31 \\
\hline Lituania & 3.15 & 0.83 \\
\hline Malasia & 2.07 & 0.63 \\
\hline Holanda & 3.49 & 0.91 \\
\hline Nueva Zelanda & 2.84 & 1.08 \\
\hline Nigeria & 1.43 & 0.89 \\
\hline Perú & 1.56 & 0.58 \\
\hline Polonia & 3.06 & 0.98 \\
\hline Portugal & 2.25 & 0.60 \\
\hline Rumania & 2.44 & 0.93 \\
\hline España & 2.80 & 0.59 \\
\hline Suecia & 3.45 & 1.35 \\
\hline Suiza & 3.24 & 0.79 \\
\hline Turquía & 3.50 & 1.12 \\
\hline Estados Unidos & 3.52 & 1.88 \\
\hline
\end{tabular}


(2.7) que los varones (0.58). En el Perú la frecuencia del llanto fue menor. En las mujeres (1.56) y en los varones (0.58). En Chile, el sexo femenino reportó que lloraba casi una vez por semana (3.6) mientras que el sexo masculino afirmó que lloraba sólo 1.22. La diferencia entre hombres y mujeres fue menor en Nigeria (Cuadro 2).

¿Con qué frecuencia creemos que lloramos?

En una escala que va de 1 (raramente lloro) a 10 (lloro con mucha frecuencia) se estimó en la población de los 30 países un promedio de 4.8 (M 5.9, V 3.3). Valores bajos se obtuvieron en Nigeria, Ghana y el Perú (Cuadro 3).

La mayor tendencia al llanto de las mujeres en los 30 países, así como el Perú se puede apreciar en la Figura 1 y Figura 2, respectivamente.

\section{Puntaje total del llanto}

Es una escala que fluctúa entre nunca (1) a siempre (7) se obtiene en la población de los 30 países un promedio de 2.6, logrando las mujeres promedio más alto (3.1) que los varones (2.1). Ambos grupos difieren más en Holanda (1.4) y menos en el Perú (0.4)

Cómo se sienten después de un episodio de llanto

Se solicitó a los sujetos que indicaran cómo se sintieron después de un episodio de llanto (relajados, tensos, depresivos, alegres). Las respuestas posibles eran (peor, igual, o mejor). Los puntajes fluctuaban entre -7 (peor) a +7 (mejor). Se constataron diferencias importantes entre los países. Suecia, Noruega y Finlandia reportaron efectos positivos, China y Nigeria efectos menores. 
El llanto del adulto

Cuadro 3

Tendencia General al Llanto

\begin{tabular}{|l|c|c|}
\hline Pais & Mujeres & Varones \\
\hline Australia & 6.00 & 3.22 \\
Austria & 6.10 & 3.66 \\
Bélgica & 5.91 & 2.92 \\
Brasil & 6.63 & 3.45 \\
Bulgaria & 5.59 & 3.58 \\
Chile & 6.40 & 3.43 \\
China & 5.28 & 3.50 \\
Finlandia & 6.57 & 3.11 \\
Ghana & 5.28 & 3.80 \\
Grecia & 5.67 & 3.11 \\
India & 5.05 & 3.23 \\
Indonesia & 5.61 & 3.06 \\
Israel & 6.28 & 2.80 \\
Italia & 6.25 & 4.46 \\
Jamaica & 6.24 & 3.18 \\
Kenya & 5.40 & 3.81 \\
Lituania & 5.90 & 2.79 \\
Malasia & 5.40 & 2.26 \\
Holanda & 6.05 & 2.66 \\
Nueva Zelanda & 5.84 & 3.50 \\
Nigeria & 4.43 & 3.49 \\
Perú & 5.40 & 3.95 \\
Polonia & 5.66 & 2.47 \\
Portugal & 6.30 & 2.81 \\
Rumania & 5.30 & 3.23 \\
España & 6.59 & 3.42 \\
Suecia & 7.02 & 3.81 \\
Suiza & 6.19 & 2.68 \\
Turquía & 6.35 & 4.04 \\
Estados Unidos & 6.34 & 3.61 \\
\hline & & \\
\hline
\end{tabular}




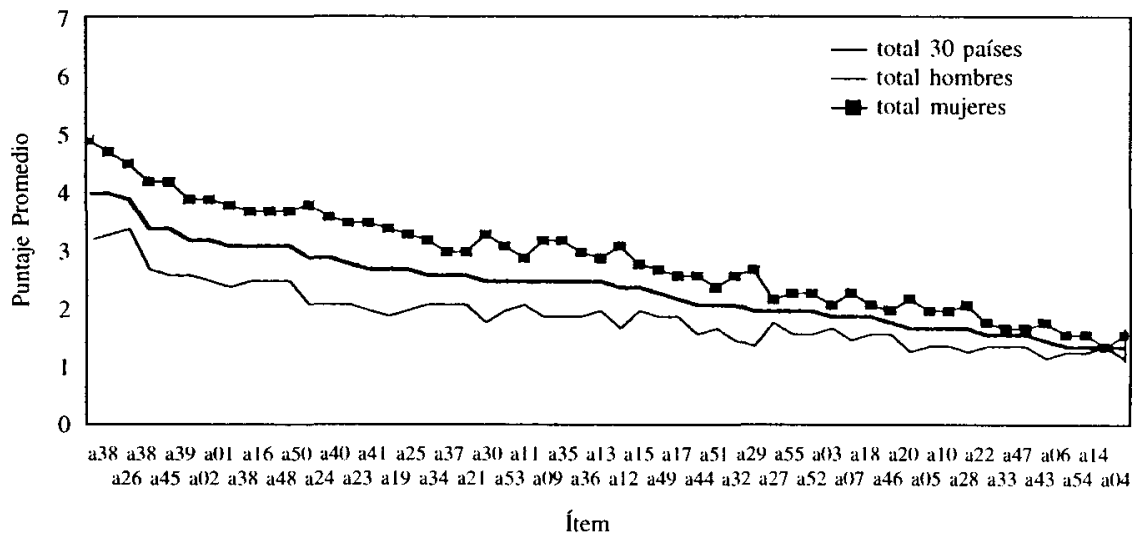

Figura 1. Frecuencia del llanto de varones y mujeres en los 30 países

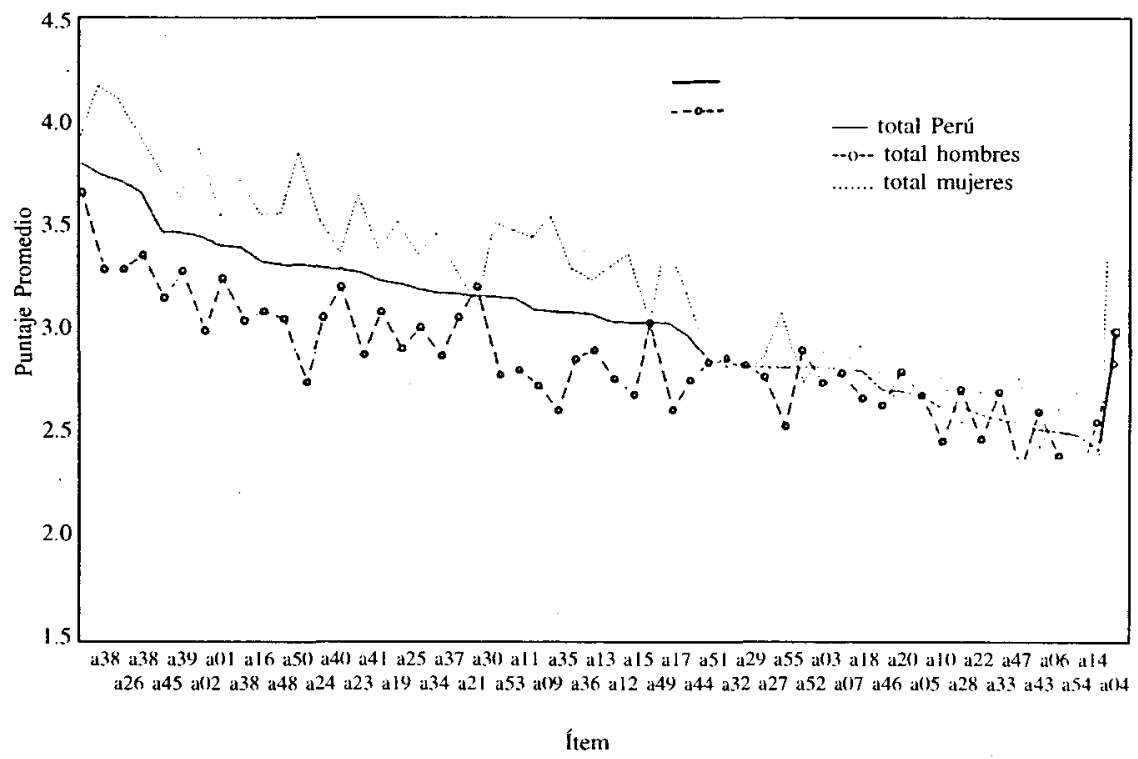

Figura 2. Frecuencia del llanto de varones y mujeres en el Perú 
El llanto del adulto

Cuadro 4

Promedio del Puntaje Total de Llanto

\begin{tabular}{|c|c|c|}
\hline pars. & Muleres & Varones \\
\hline Australia & 3.4 & 2.0 \\
\hline Austria & 2.9 & 2.1 \\
\hline Bélgica & 3.0 & 2.0 \\
\hline Brasil & 3.3 & 2.1 \\
\hline Bulgaria & 2.7 & 2.0 \\
\hline Chile & 3.1 & 2.1 \\
\hline China & 3.4 & 2.5 \\
\hline Finlandia & 3.3 & 2.1 \\
\hline Ghana & 3.0 & 2.3 \\
\hline Grecia & 3.2 & 1.9 \\
\hline India & 2.8 & 2.2 \\
\hline Indonesia & 3.2 & 2.2 \\
\hline Israel & 3.0 & 1.7 \\
\hline Italia & 3.0 & 2.0 \\
\hline Jamaica & 3.1 & 2.3 \\
\hline Kenya & 3.3 & 2.7 \\
\hline Lituania & 2.8 & 1.8 \\
\hline Malasia & 3.0 & 1.8 \\
\hline Holanda & 3.3 & 1.9 \\
\hline Nueva Zelanda & 3.1 & 2.1 \\
\hline Nigeria & 3.1 & 2.6 \\
\hline Perú & 3.1 & 2.7 \\
\hline Polonia & 2.9 & 1.7 \\
\hline Portugal & 2.8 & 2.0 \\
\hline Rumania & 2.6 & 1.7 \\
\hline España & 3.3 & 2.4 \\
\hline Suecia & 3.4 & 2.2 \\
\hline Suiza & 2.8 & 2.0 \\
\hline Turquía & 3.2 & 2.3 \\
\hline Estados Unidos & 3.2 & 2.3 \\
\hline
\end{tabular}




\section{Cuadro 5}

Promedio de Puntajes de la Escala de Cambio de Ánimo

\begin{tabular}{|l|c|c|c|}
\hline Grupo muresral & $\bar{X}$ & $D . E$ & $n$ \\
\hline Varones & 3.23 & 3.00 & 1507 \\
Mujeres & 3.54 & 2.95 & 2124 \\
Australia & 2.77 & 3.45 & 232 \\
Austria & 4.32 & 2.66 & 79 \\
Bélgica & 3.46 & 2.90 & 198 \\
Brasil & 3.60 & 2.48 & 96 \\
Bulgaria & 2.80 & 2.81 & 101 \\
Chile & 4.23 & 2.40 & 103 \\
China & 1.94 & 2.24 & 69 \\
Finlandia & 4.70 & 2.31 & 104 \\
Ghana & 3.60 & 2.89 & 83 \\
Grecia & 3.62 & 2.65 & 113 \\
India & 3.29 & 3.21 & 241 \\
Indonesia & 3.00 & 3.11 & 157 \\
Israel & 2.76 & 3.09 & 97 \\
Italia & 3.06 & 2.87 & 111 \\
Jamaica & 3.48 & 3.39 & 87 \\
Kenya & 4.03 & 3.34 & 163 \\
Lituania & 4.05 & 2.40 & 186 \\
Malasia & 3.10 & 2.96 & 84 \\
Holanda & 4.32 & 2.35 & 159 \\
Nueva Zelanda & 2.53 & 2.91 & 75 \\
Nigeria & 2.29 & 3.61 & 71 \\
Perú & 2.51 & 4.42 & 43 \\
Polonia & 2.98 & 2.75 & 103 \\
Portugal & 3.79 & 2.51 & 122 \\
Rumania & 3.32 & 3.05 & 154 \\
España & 3.70 & 2.59 & 101 \\
Suecia & 4.54 & 2.27 & 94 \\
Suiza & 3.13 & 3.11 & 99 \\
Turquía & 3.29 & 2.77 & 99 \\
Estados Unidos & 2.95 & 3.15 & 207 \\
\hline Total & 3.41 & 2.92 & 3631 \\
\hline
\end{tabular}


El llanto del adulto

\section{Parte D}

\section{Último episodio de llanto}

El Cuadro 6 enumera las situaciones y emociones que inducen al llanto, siendo las más importantes los conflictos, sufrimientos e inadecuación personal.

\section{Cuadro 6}

Frecuencia de las Causas del Último Episodio de Llanto en Porcentajes

\begin{tabular}{|l|c|c|c|}
\hline Causa & & \%o & \\
\hline & $\begin{array}{r}\text { Varones } \\
(952)\end{array}$ & $\begin{array}{c}\text { Mujeres } \\
(1527)\end{array}$ & $\begin{array}{c}\text { Total } \\
(2479)\end{array}$ \\
\hline Conflicto & 15 & 23 & 20 \\
Pérdida & 30 & 24 & 26 \\
Inadecuación personal & 0 & 12 & 12 \\
Observar sufrimiento & 18 & 14 & 15 \\
Observar eventos positivos & 14 & 6 & 9 \\
Estados físicos & 2 & 3 & 3 \\
Estados psicológicos & 8 & 10 & 9 \\
Combinación de causas & 3 & 6 & 5 \\
Otros & 1 & 2 & 2 \\
\hline
\end{tabular}

¿Cuándo ocurrió el último episodio?

Con mayor frecuencia, las mujeres indicaron que han tenido episodios de llanto más recientes que los varones. Ellos informan que el último episodio de llanto ocurrió hace más de 6 meses; en el sexo femenino, se ha dado esa situación sólo en un 5\%. 


\section{Cuadro 7}

¿Hace Cuánto Ocurrió el Último Episodio de Llanto?

\begin{tabular}{|c|c|c|c|c|}
\hline \multirow{2}{*}{ Periodo } & \multicolumn{4}{|c|}{$\frac{0.9 \%}{30 \text { paises }}$} \\
\hline & $\begin{array}{l}\text { Warones } \\
\text { (1608) }\end{array}$ & $\begin{array}{c}\text { Mujeres } \\
(2261)\end{array}$ & $\begin{array}{l}\text { Yarones } \\
(45)\end{array}$ & $\begin{array}{c}\text { Mujeres } \\
(48)\end{array}$ \\
\hline Menos de 1 día & 4 & 12 & 2.2 & 6.3 \\
\hline 2 - 5 días atrás & 9 & 28 & 8.9 & 14.6 \\
\hline 5 - 10 días atrás & 11 & 19 & 8.9 & 16.7 \\
\hline 10 - 30 días atrás & 19 & 22 & 15.6 & 14.6 \\
\hline $1-6$ meses atrás & 28 & 14 & 26.7 & 20.8 \\
\hline $7-12$ meses atrás & 一 & - & 15.6 & 22.9 \\
\hline
\end{tabular}

\section{Duración}

Las mujeres reportaron que el llanto duró más tiempo que los hombres; aunque, en general, ambos grupos indicaron que el llanto duró menos de 5 minutos (Cuadro 8).

\section{Cuadro 8}

Duración del Último Episodio de Llanto en Porcentajes

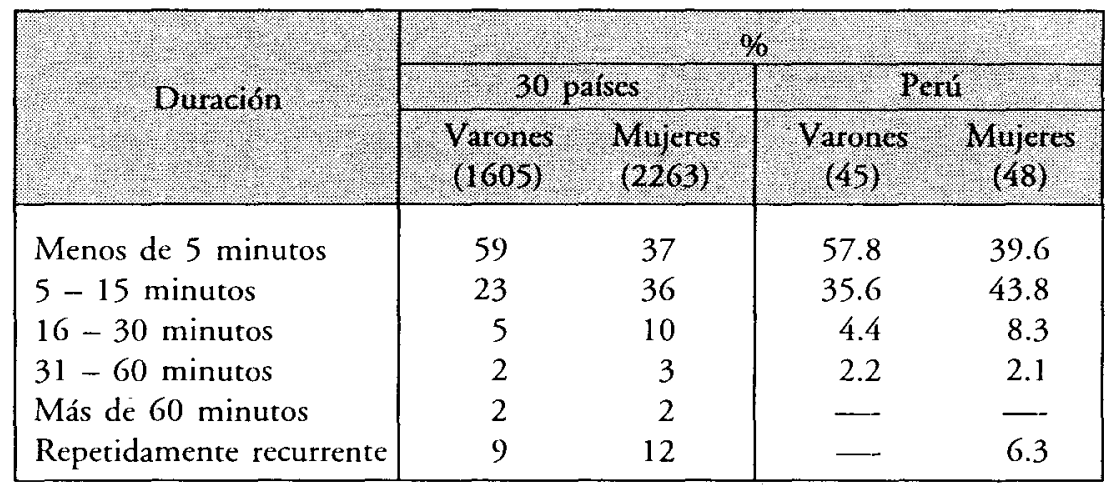


El llanto del adulto

\section{Intensidad}

Las mujeres tienden a tener un llanto más intenso que los varones. Estos últimos informaron que sólo se les humedeció los ojos, sin otro componente conductual (Cuadro 9).

Cuadro 9

Intensidad del Llanto en Porcentajes

\begin{tabular}{|c|c|c|c|c|}
\hline \multirow{3}{*}{ lat } & \multicolumn{4}{|c|}{ 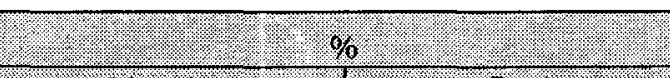 } \\
\hline & \multicolumn{2}{|c|}{30 paises: } & \multicolumn{2}{|c|}{ Pera } \\
\hline & $\begin{array}{l}\text { Yarones } \\
(1607)\end{array}$ & $\begin{array}{l}\text { Mujeres } \\
(2265)\end{array}$ & $\begin{array}{l}\text { Yarones } \\
(45)\end{array}$ & $\begin{array}{c}\text { Mujeres } \\
(48)\end{array}$ \\
\hline $1=$ ojos húmedos & 47 & 27 & 42.2 & 16.7 \\
\hline $2=1+$ Sollozo silencioso & 37 & 52 & 40.0 & 62.5 \\
\hline $3=2+$ gritos & 9 & 13 & 13.3 & 14.6 \\
\hline $\begin{array}{l}4=3+\text { movimientos } \\
\text { corporales }\end{array}$ & 8 & 8 & 4.4 & 6.3 \\
\hline
\end{tabular}

\section{Lugar en el que ocurrió el llanto}

El $75 \%$ de la muestra afirmó haberse encontrado en el hogar durante el episodio de llanto (Cuadro 10).

Cuadro 10

\section{Lugar del Llanto en Porcentajes}

\begin{tabular}{|c|c|c|c|}
\hline \multirow{3}{*}{ Lugar } & \multicolumn{3}{|c|}{$0 \%$} \\
\hline & \multicolumn{2}{|c|}{ To parses: } & Pera \\
\hline & $\begin{array}{l}\text { Varones } \\
(111.15)\end{array}$ & $\begin{array}{l}\text { Mujeres } \\
(1625) \\
\end{array}$ & $\begin{array}{l}\text { Varones } \\
(2740)\end{array}$ \\
\hline En la casa & 71 & 78 & 75 \\
\hline En la escuela & 3 & 4 & 4 \\
\hline En el camino & 9 & 8 & 8 \\
\hline En el trabajo & 2 & 2 & 2 \\
\hline En los servicios médicos & 2 & 1 & 1 \\
\hline En lugares públicos & 13 & 8 & 10 \\
\hline
\end{tabular}


¿A qué hora ocurrió el llanto?

El $49 \%$ de los participantes reportó que el llanto ocurrió entre las 6 p.m. y 12 p.m. (Cuadro 11).

Cuadro 11

Hora de Ocurrencia del Llanto en Porcentajes

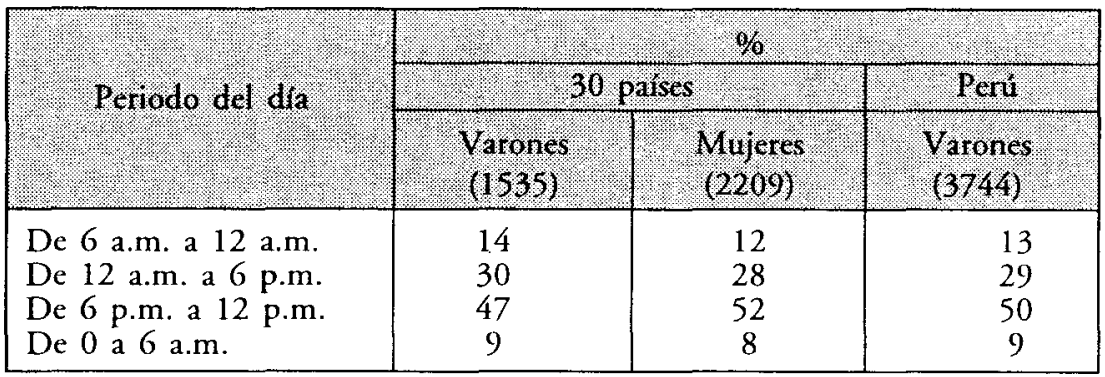

\section{Persona responsable del llanto}

Se encontró que, tanto los hombres como las mujeres, atribuyen su llanto a su pareja, a un familiar o a un amigo íntimo (Cuadro 12).

Cuadro 12

Responsable de la Situación en Porcentajes

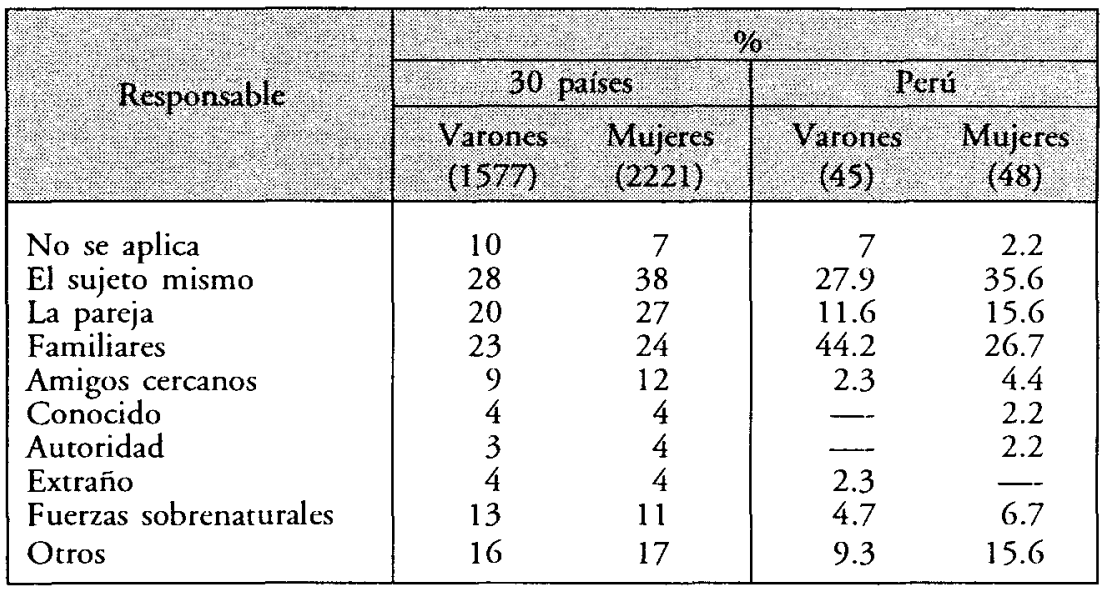


El llanto del adulto

Sentimientos que se expresan a través del llanto

Los sujetos experimentan variedad de sentimientos, siendo los más frecuentes la tristeza, la impotencia, la cólera y la frustración (Cuadro 13).

Cuadro 13

Emociones que Experimentó en Porcentajes

\begin{tabular}{|c|c|c|c|c|}
\hline \multirow{2}{*}{ Emocion } & \multicolumn{4}{|c|}{$\frac{30 \text { parses }}{30}$} \\
\hline & $\begin{array}{l}\text { Varones } \\
\text { V. } \\
(11603)\end{array}$ & $\begin{array}{c}\text { Mujeres } \\
(2257)\end{array}$ & $\begin{array}{c}\text { Varones } \\
(45)\end{array}$ & $\begin{array}{c}\text { Mujeres: } \\
(48)\end{array}$ \\
\hline No sé & 6 & 4 & 4.4 & - \\
\hline Alivio & 15 & 14 & 6.7 & 8.3 \\
\hline Alegría & 12 & 6 & 6.7 & 6.3 \\
\hline Desprecio & 8 & 10 & 8.9 & 10.4 \\
\hline Tristeza & 55 & 65 & 53.3 & 43.8 \\
\hline Temor & 12 & 19 & 11.1 & 10.4 \\
\hline Humillación & 9 & 14 & 4.4 & 12.5 \\
\hline Autocompasión & 16 & 25 & 8.9 & 14.6 \\
\hline Impotencia & 32 & 44 & 35.6 & 41.7 \\
\hline Satisfacción & 8 & 4 & - & - \\
\hline Cólera & 22 & 32 & 15.6 & 14.6 \\
\hline Disgusto & 10 & 12 & 11.1 & 22.9 \\
\hline Culpa & 14 & 16 & 11.1 & 6.3 \\
\hline Gozo & 6 & 4 & 2.2 & 6.3 \\
\hline Frustración & 24 & 31 & 22.2 & 25.0 \\
\hline Congoja & 14 & 18 & 24.4 & 16.7 \\
\hline Ser afectado & 22 & 19 & 15.6 & 27.1 \\
\hline Éxtasis & 5 & 2 & 4.4 & 2.1 \\
\hline Otros & 9 & $\cdot 12$ & 2.2 & 6.3 \\
\hline
\end{tabular}

¿El llanto cambió la relación con las personas implicadas?

Ambos sexos reportaron efectos positivos, aunque, en general, sintieron que el llanto no tuvo mayor efecto en sus relaciones con los demás (Cuadro 14). 


\section{Cuadro 14}

Cambio en las Relaciones con Otros en Porcentajes

\begin{tabular}{|c|c|c|c|c|}
\hline \multirow{3}{*}{ Cambio: } & \multicolumn{4}{|c|}{ 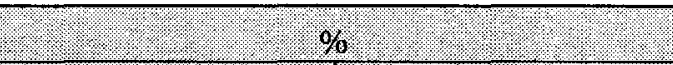 } \\
\hline & \multicolumn{2}{|c|}{1.30 paises. } & \multicolumn{2}{|c|}{ Peru } \\
\hline & $\begin{array}{l}\text { Yarones } \\
(9211)\end{array}$ & $\begin{array}{l}\text { Majeres } \\
(1370)\end{array}$ & $\begin{array}{c}\text { Varones } \\
(44)\end{array}$ & $\begin{array}{c}\text { Migeres } \\
(48)\end{array}$ \\
\hline Empeoró & 9 & 9 & 4.5 & 6.4 \\
\hline Igual & 67 & 63 & 79.5 & 82.6 \\
\hline Mejoró & 25 & 28 & 15.9 & 10.6 \\
\hline
\end{tabular}

¿Por qué las personas dejan de llorar?

Las personas, generalmente, sienten que se han estabilizado, que ya era suficiente, que habían superado el problema o que se habían sentido confortadas o apoyadas por alguien (Cuadro 15).

Cuadro 15

Motivos para Parar de Llorar en Porcentajes

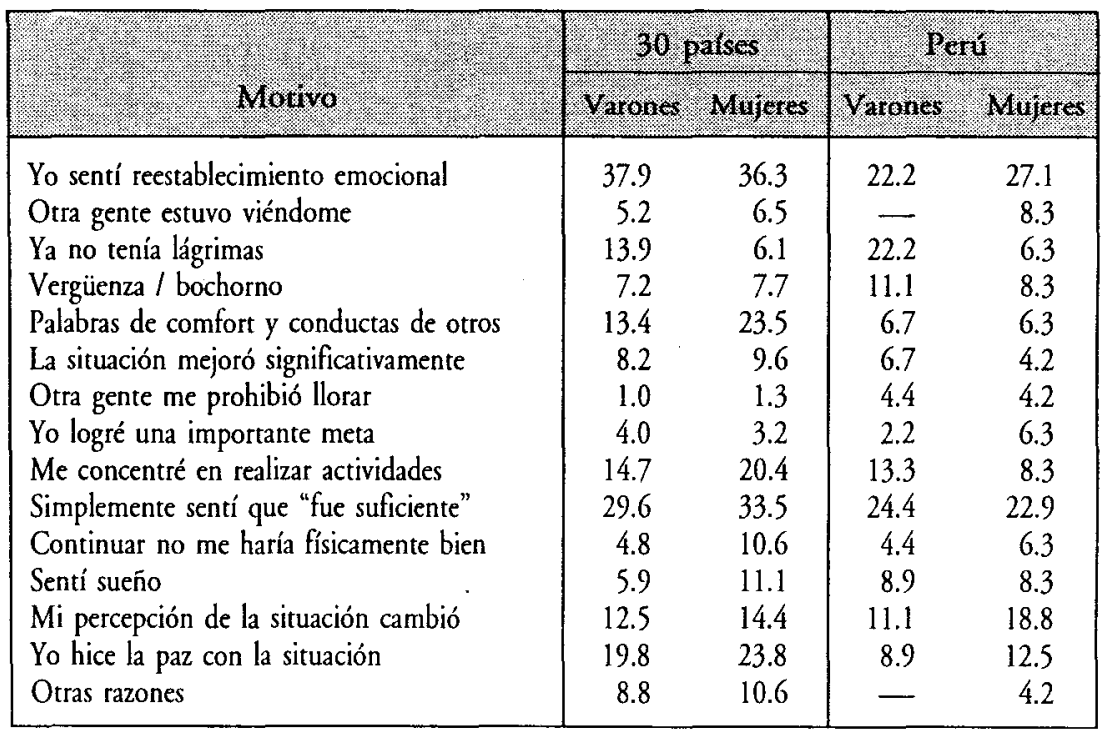


El llanto del adulto

\section{Parte E}

\section{Efecto del ciclo menstrual en la tendencia al llanto}

El $43 \%$ de las mujeres indicaron que había una relación entre el ciclo menstrual y el llanto. Se observaron diferencias importantes, mientras que $69.2 \%$ de la mujeres turcas mantenían la relación indicada, sólo lo hacen $15 \%$ de las mujeres chinas (Cuadro 16).

Cuadro 16

Mujeres que Reportan Relación entre el Llanto y el Ciclo Menstrual en Porcentajes

\begin{tabular}{|l|c|}
\hline Pais & \% de Mujeres \\
\hline Australia & 68.9 \\
Austria & 40.0 \\
Bélgica & 25.1 \\
Brasil & 55.8 \\
Bulgaria & 35.3 \\
Chile & 65.5 \\
China & 15.4 \\
Finlandia & 63.3 \\
Ghana & 18.9 \\
Grecia & 49.4 \\
India & 29.5 \\
Indonesia & 40.7 \\
Israel & 36.0 \\
Italia & 32.9 \\
Jamaica & 29.7 \\
Kenya & 61.0 \\
Lituania & 32.6 \\
Malasia & 29.2 \\
Holanda & 61.5 \\
Nueva Zelanda & 46.9 \\
Nigeria & 22.0 \\
Perú & 14.6 \\
Polonia & 32.1 \\
Portugal & 31.3 \\
Rumania & 19.8 \\
España & 64.7 \\
Suecia & 59.2 \\
Suiza & 50.7 \\
Turquía & 69.2 \\
Estados Unidos & 58.8 \\
\hline Total & 44.9 \\
\hline
\end{tabular}




\section{Relación entre las variables}

Se correlacionaron las variables más importantes, observándose que la frecuencia del llanto correlaciona 0.42 con el Puntaje Total de Llanto y 0.55 con la Tendencia al Llanto. Por otro lado, el Puntaje Total del Llanto correlaciona 0.63 con la Tendencia al Llanto. El patrón de las correlaciones apoya la validez del cuestionario respecto a los datos de frecuencia.

\section{Conclusiones}

1. Las mujeres lloran con más frecuencia que los varones. Sus episodios de llanto son más intensos y de mayor duración.

2. Se observan diferencias importantes entre los países en relación a la frecuencia y tendencia del llanto.

3. Los conflictos, pérdidas, sufrimiento e inadecuación personal son las causas más frecuentes que inducen el llanto.

4. En cuanto a otras variables la semejanza entre $V$ y $M$ es mayor que las diferencias.

5. Los sujetos experimentan durante el llanto sentimientos de tristeza, de impotencia, frustración y cólera.

6. Después del llanto generalmente $V$ y $M$ se sienten mejor si logran mejorar sus relaciones o la situación

7. Se constataron diferencias significativas entre los países en cuanto a la relación entre el ciclo menstrual y tendencia al llanto.

8. Es importante destacar, que según se observa en los Cuadros presentados, los resultados obtenidos en el Perú, con una muestra de 93 sujetos, tienden generalmente, a coincidir con los obtenidos en los 30 países.

\section{Referencias}

Cornelius, R.R. (1981). Weeping as a social interaction: The interpersonal logic of moist eye. Dissertation Abstracts International, 42 (08), 3491 - 
El llanto del adulto

3492.

Cornelius, R.R. (1986, April). Prescience in the pre-scientific study of weeping? $A$ history of weeping in the popular press from the mid-1800's to the present. Ponencia presentada en el 57 Encuentro Anual de la Asociación Psicológica del Este, Nueva York.

Frey, W. H.(1985). Crying: The mystery of tears. Minneapolis: Winston Press.

Frijda, N.H. (1997). On the functions of emotional expression. En A.J.J.M. Vingerhoets, F.J. Van Bussel y A.J.W. Boelhouwer (Eds.), The (non) expression of emotions en health and disease. Tilburg: Tilburg University Press.

Kottler, J.A.(1996). The language of tears. San Francisco: Jossey-Bass.

Labott, S.M. y Martin, R.B. (1987). The stress-moderating effects of weeping and humor. Journal of Human Stress, 13, 159-164.

Scherer, K.R., Summerfield, A.B. y Wallbott, H.G. (1983). Cross-national research on antecedents and components of emotion: A progress report. Social Science Information, 22, 355-385.

Vingerhoets, A.J.J.M., Van den Berg, M.., Kortekas, R. Th., Van Heck, G.I. y Croon, M. (1993). Weeping: Association with personality, coping, and subjective health status. Personality and Individual Differences, 14. 185-190

Vingerhoets, A.J.J.M., Van Geleuken, A.J.M.L., Van Tiburg, M.A.L. y Van Heck, G.L. (1997). The psychological context of crying episodes: Toward a model of adult crying. En A.J.J.M. Vingerhoets, F.J. Van Bussel, y A.J.W. Boelhouwer (Eds.), The (non) expression of emotions in health and disease. Tilburg: Tilburg University Press.

Vingerhoets, A.J.J.M., y Becht, M.C. (1997). International Study on Adult Crying: Some first result. Poster presentado en el Encuentro Anual de la Sociedad Psicosomática Americana, Santa Fe, NM. 


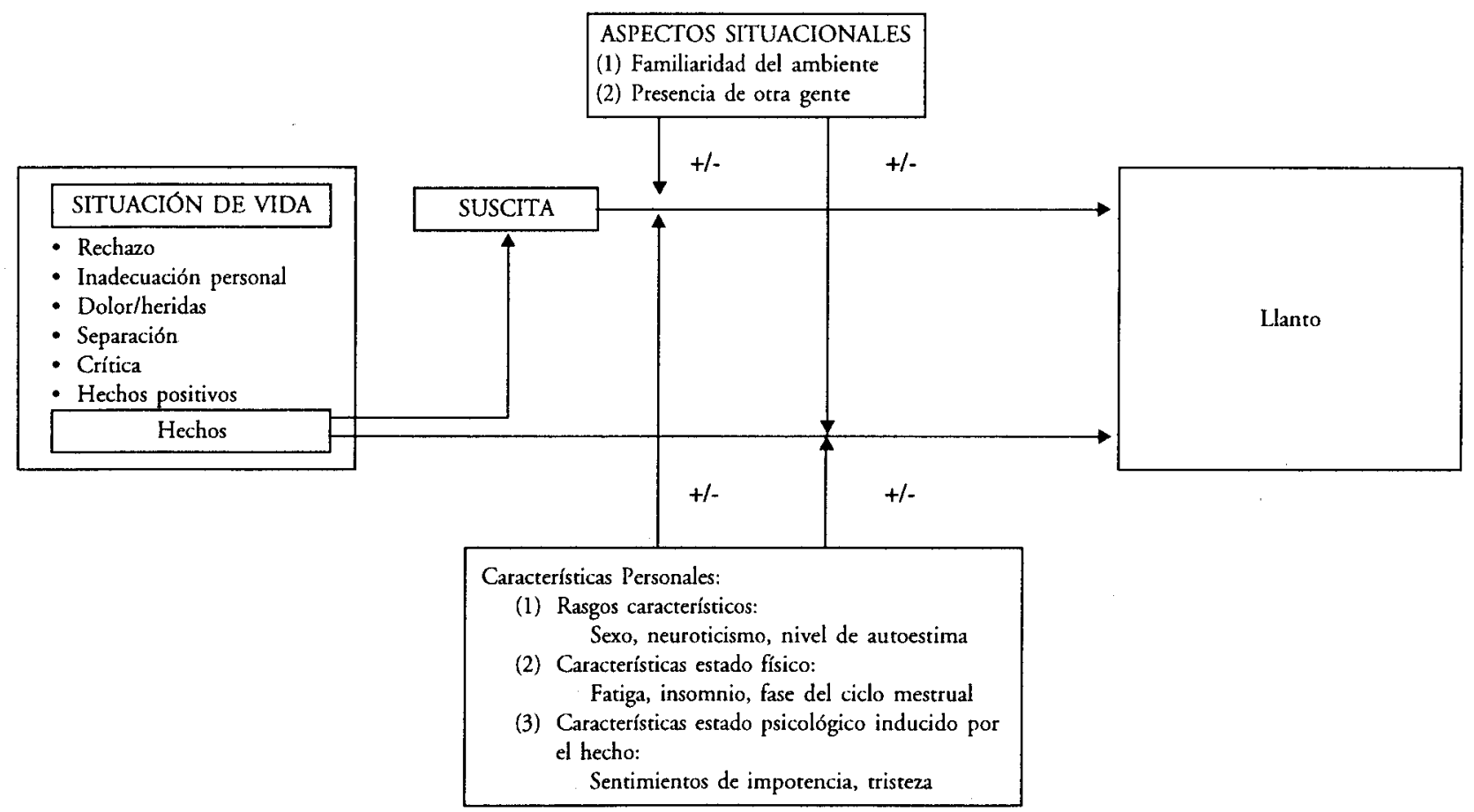

Figura A1. Modelo del llanto de los adultos 